www.jmscr.igmpublication.org Impact Factor 5.244

Index Copernicus Value: 83.27 ISSN (e)-2347-176x ISSN (p) 2455-0450 crossref DOI:_https://dx.doi.org/10.18535/jmscr/v4i12.110

\title{
Role of Duplex Ultrasonography in the Evaluation of Portal Venous Hypertension
}

\author{
Authors \\ Rajesh Pattanaik ${ }^{1}$, Bararuchi Dash ${ }^{2}$, Shamimun Nisa ${ }^{2}$, Braja Behari Panda $^{2}$, \\ Savitri Bhagat ${ }^{3}$, Soumya Ranjan Nayak ${ }^{1}$ \\ ${ }^{1}$ Jr. Resident, ${ }^{2}$ Associate Professor, ${ }^{3}$ Professor \\ Department of Radiodiagnosis, VSS Institute of Medical Sciences and Research, Burla, Odisha, India \\ Corresponding Author \\ Rajesh Pattanaik \\ Email: rajesh.pattanaik@gmail.com, Contact-9437725051
}

\begin{abstract}
Aims \& Objectives: The objective of our study is to know the spectrum of colour Doppler and ultrasonographic findings in portal hypertension \& to assess the value of duplex ultrasonography to diagnose \& establish the cause of portal hypertension.

Materials \& Methods: A Cross-sectional study was conducted from December 2014 to November 2016 on 100 clinically suspected / diagnosed cases of portal hypertension, who were referred to our department. All the patients underwent $2 D$ grey scale \& colour doppler ultrasonography of abdomen and pelvis using a curvilinear probe of 3.5 - 5.0 MHZ. Analysis of Percentage and proportions of various morphologic \& hemodynamic parameters \& etiology of portal hypertension were done.

Results: Among 100 cases of portal hypertension who were studied, $72 \%$ were males \& $59 \%$ in age group of 40 to 59 yrs. Most common etiology of portal hypertension was Cirrhosis (71\%). Portal vein diameter $>13 \mathrm{~mm}$ was seen in $61 \%$ of cases. Loss of respiratory phasicity of portal vein was seen in $84 \%$ cases. Decreased portal vein velocity was noted in $44 \%$ cases. Congestion index $>0.1$ seen in $92 \%$ of cases. Hepatopetal, hepatofugal and bidirectional to and fro flow was noted in 78\%, 4\% and 3\% respectively. Among Porto-systemic collaterals was noted in $92 \%$ of the cases most common being spleno-renal and gastro-renal collaterals, seen in $87 \%$ of cases. Splenomegaly and ascites were seen in $93 \%$ and $85 \%$ cases respectively.
\end{abstract}

Conclusion: Portal hypertension is a commonly encountered clinical condition with multiple causes and several sequelae. Ultrasound is an accurate non-invasive tool giving significant hemodynamic information and helps in assessing etiology, severity and complications of Portal Hypertension.

Keywords- Portal Hypertension, duplex ultrasonography, cirrhosis, Porto-systemic collaterals.

\section{INTRODUCTION}

Portal hypertension is a common clinical syndrome, characterised by a sustained increase in portal venous pressure. It is defined as a wedged hepatic vein pressure or direct portal vein pressure of more than $5 \mathrm{mmHg}$ greater than the inferior vena cava pressure, a splenic vein pressure of greater than 15 $\mathrm{mm} \mathrm{Hg}$ or surgically measured portal venous pressure of greater than $30 \mathrm{~cm}$ water ${ }^{[1]}$. 
Portal hypertension syndrome is a common evolutive complication of several hepatic and extrahepatic diseases, liver cirrhosis responsible for more than $80 \%$ of cases. ${ }^{[2]}$ When diagnosed it has prognostic value because of the high incidence of hemorrhagic, metabolic and infectious complications that these patients may develop, so clinical suspicion must be confirmed by objective complementary studies that provide information about the etiology and severity of the disease there by helps in timely implementation of surgical or medical management and thus prevent complication.

However, actual direct measurements of portal pressures are obtained in only a small minority of patients. Consequently, non-invasive imaging modalities and in particular ultrasound, play a crucial role in the diagnosis and management of portal hypertension.

\section{AIMS AND OBJECTIVES}

$>$ To evaluate the spectrum of duplex Ultrasonography findings in portal hypertension.

To assess the value of duplex Ultrasonography in the diagnosis of portal hypertension.

\section{MATERIALS AND METHODS}

Source of data: This study is a hospital based study at Veer Surendra Sai Institute of Medical Sciences and Research (VIMSAR), Burla, Sambalpur, Odisha.

Type of study: Cross sectional study.

Sample: 100 patients were selected for our study randomly from the patients referred to the department of radiodiagnosis with the clinical suspicion / clinical diagnosis of portal hypertension, in the period of 2 years i.e. from December 2014 to November 2016.

Inclusion criteria: All cases with clinical suspicion of portal hypertension.

\section{Exclusion criteria:}

1. Patients who underwent hepatobiliary surgery or recent surgery for any other reasons.

2. Trauma and pregnant patients.

Procedure: After taking informed consent, all patients included in the study underwent 2D grey scale and colour Doppler ultrasonography of abdomen using a curvilinear probe of 3.5 - 5.0 MHZ coupled with colour Doppler equipment (Philips HD7 ultrasound machine), and morphology and hemodynamic parameters of portal hypertension were assessed. Statistical analysis was done using percentage and proportions.

\section{RESULTS}

Total 100 patients were evaluated, most common etiology [Fig-1] was found to be cirrhosis [Fig-2] from various causes seen in 71 cases $(71 \%)$. Portal vein occlusion was seen in 17 cases not having cirrhosis of liver, out of which 7 cases were due to malignancy.

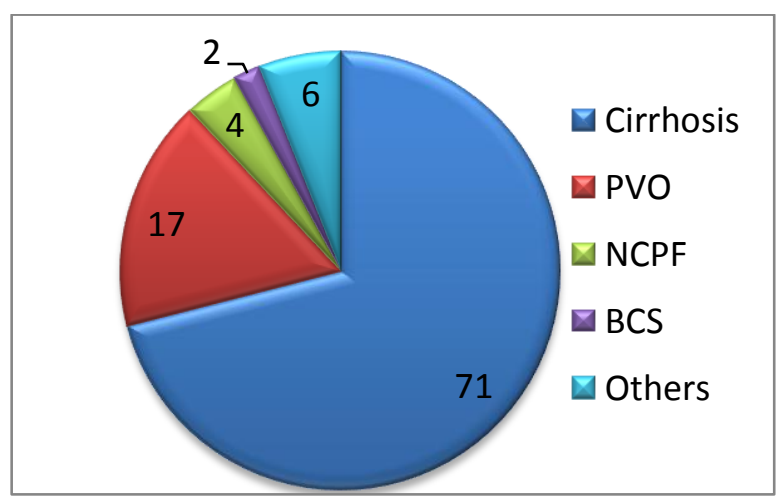

Figure 1-Etiology of portal hypertension

No etiology could be determined by ultrasound in only $6 \%$ cases, one of which was found to be a case of segmental agenesis of segment $5 \& 8$ of liver, associated with portal hypertension [Fig-10]. Males were affected more than females, $72 \%$ males as against $28 \%$ females [Fig-3], with a male to female ratio of $2.6: 1$ and $59 \%$ of cases were in the age group of 40-59 years. 


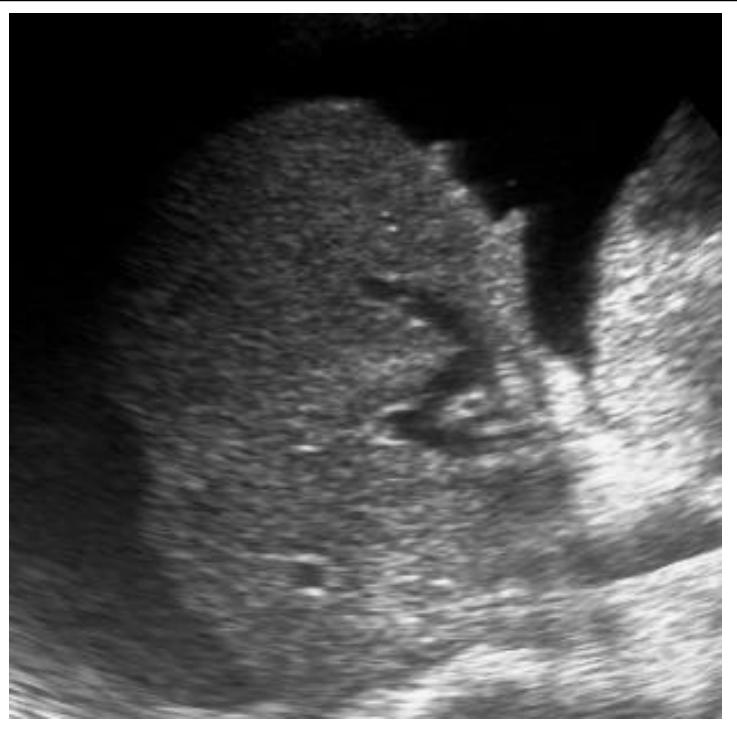

Figure 2- Cirrhosis of liver, gross ascites.

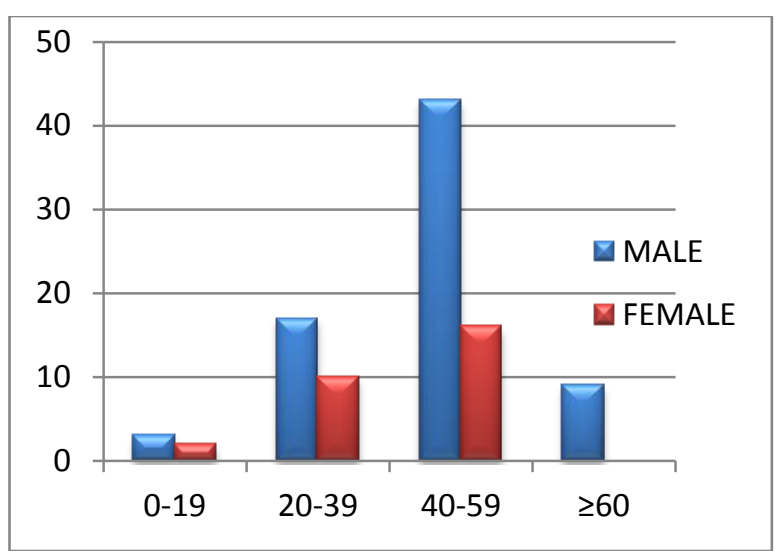

Figure 3-Age \& sex distribution

Portal vein diameter of $>13 \mathrm{~mm}$ was noted in 58 out of 95 portal hypertension patients (61\%) [Fig-4]. Diameter of portal vein could not be measured in 5 cases where portal vein was not delineated due to cavernoma formation. Increase in portal vein diameter less than $20 \%$ with deep inspiration noted in 80 out of 95 patients i.e. $84 \%$ cases. Using a mean portal vein velocity cutoff of $15 \mathrm{~cm} / \mathrm{sec}, 44 \%$ cases showed decreased value, $37 \%$ cases had increased velocity, 19\% showed no/ hepatofugal flow.

Congestion Index (CI) of portal vein is calculated as ratio of Cross-sectional area of the portal vein (in $\mathrm{cm}^{2}$ ) and Mean flow velocity in Portal Vein (in $\mathrm{cm} / \mathrm{sec}$ ). CI value of $\geq 0.1$ is significant for diagnosing portal hypertension. In 19 cases CI was Indeterminate or negative as no / reversal flow in portal vein due to complete thrombus, hepato-fugal flow and portal cavernoma. Out of remaining 81 patients, $92.5 \%$ of case showed CI of portal vein $\geq$ 0.1 . The direction of flow was hepatopetal in majority (78\%) of the cases and hepatofugal flow was in only 4 cases. Bidirectional flow was noted in 3 cases and no flow due to complete thrombus was noted in 10 cases, 5\% cases developed cavernoma with indistinguishable Portal vein.

Thrombosis in portal vein [Fig-6] observed in $18 \%$ with 10 cases showing complete thrombus. 8\% cases showed thrombus in splenic vein. Portosystemic collaterals [Fig-5] were visualised in $92 \%$ of the cases. Most frequent collateral were the Splenorenal [Fig-7] and gastro renal collaterals which were seen in $87 \%$ cases. Other visualised collaterals included GE junction varices (56\%) [Fig$8,9]$, recanalised paraumbilical vein $(42 \%)$, GB wall collaterals $(10 \%) \&$ portal cavernoma $(5 \%)$. Splenomegaly and ascites were seen in $93 \%$ and $85 \%$ cases respectively.

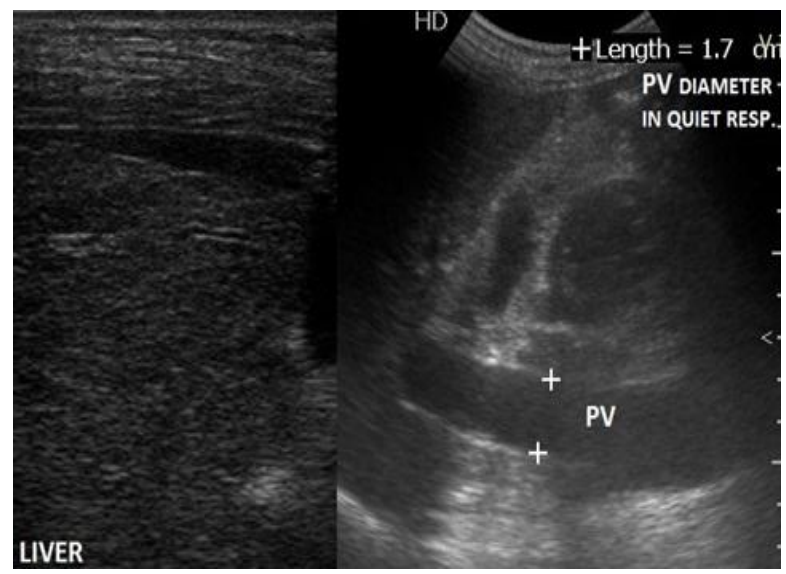

Figure 4-Liver cirrhosis, enlarged portal vein

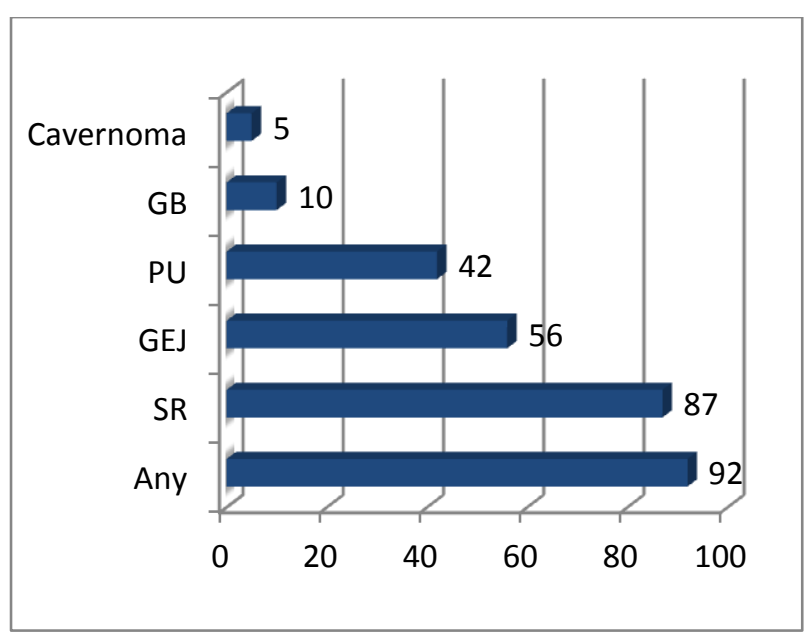

Figure 5- Collateral veins seen 


\section{JMSCR Vol||04||Issue||12||Page 14973-14980||December}

\section{6}
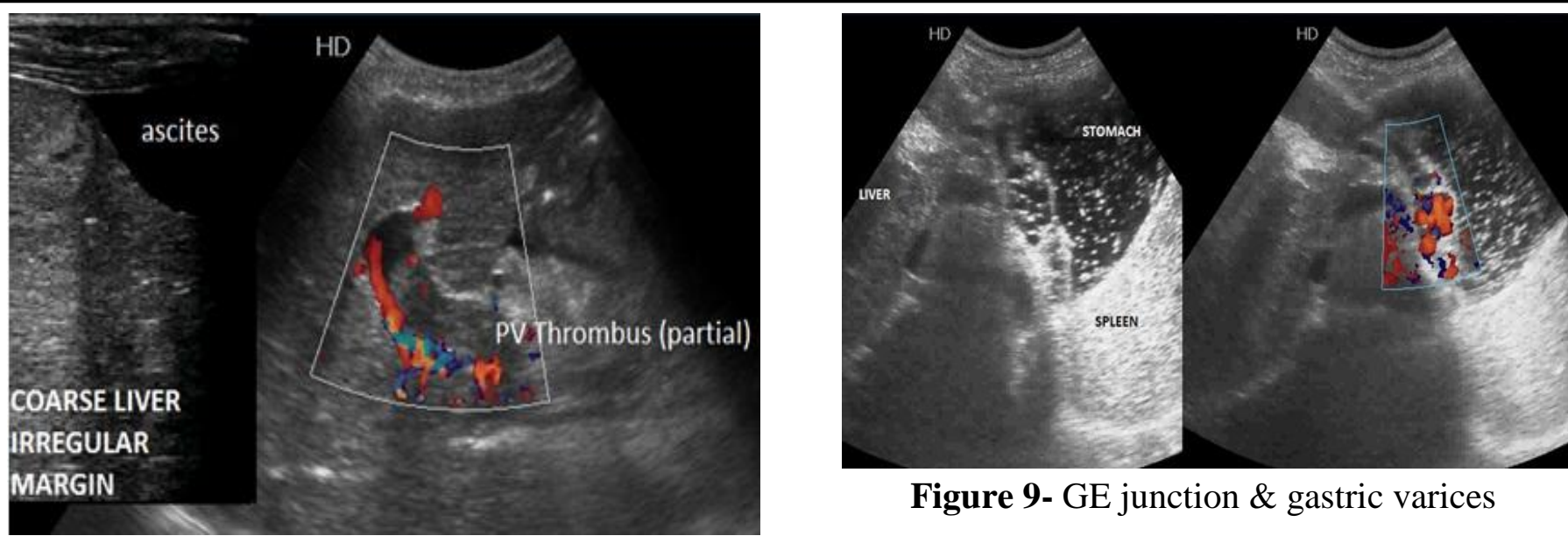

Figure 9- GE junction \& gastric varices

Figure 6- Cirrhosis with partial portal vein thrombus

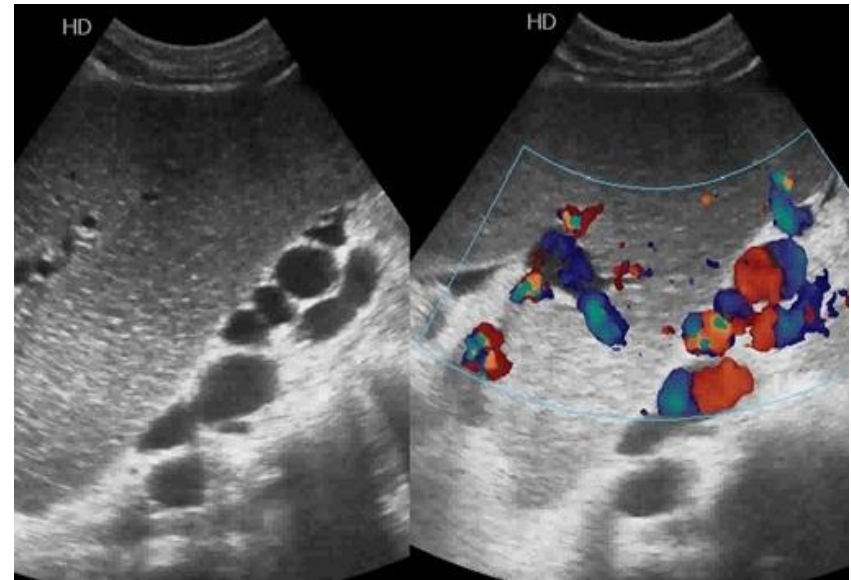

Figure 7- Splenomegaly with splenorenal collaterals

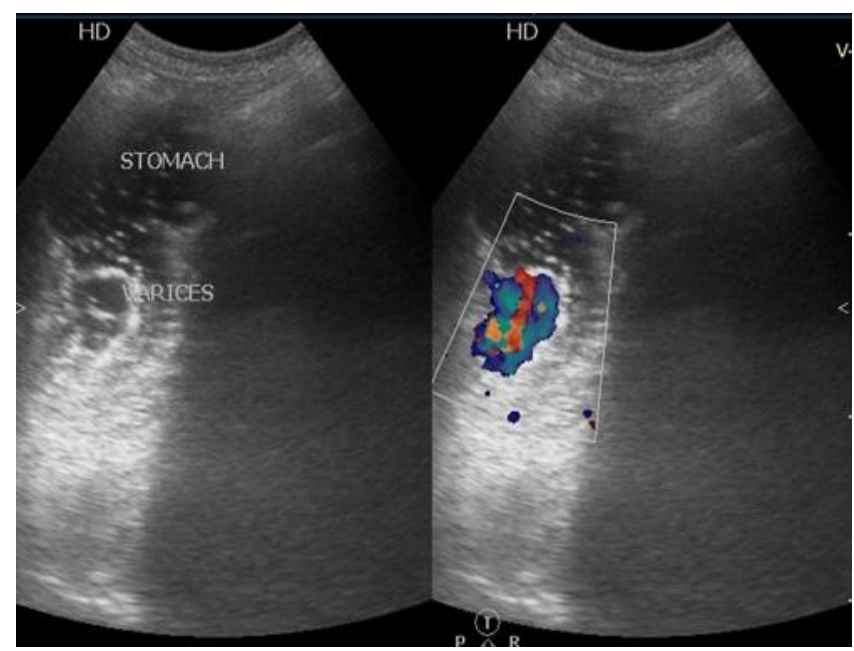

Figure 8-GE junction varices

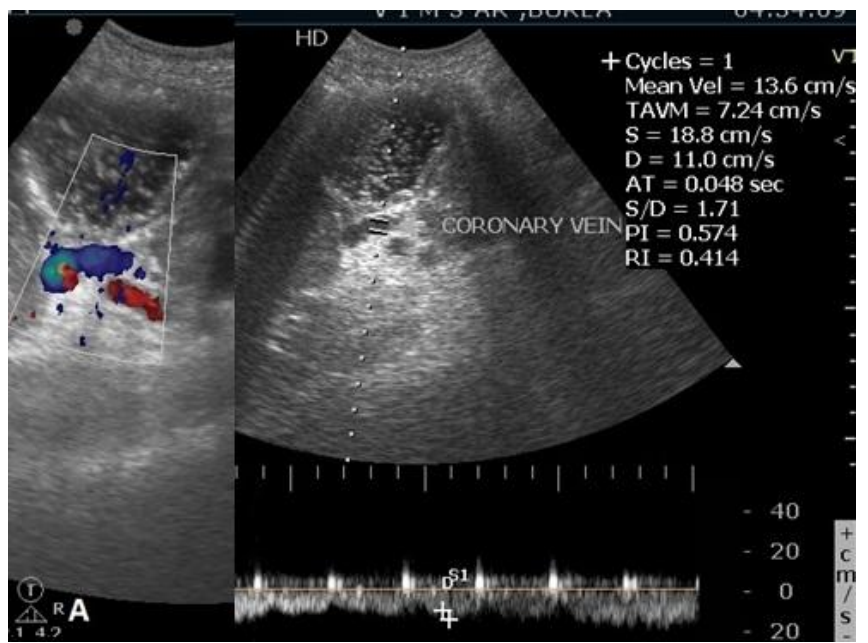

Figure 10-Dilated Coronary Vein with Continuous Hepatofugal Flow (PSV- $18.8 \mathrm{~cm} / \mathrm{s}$ )

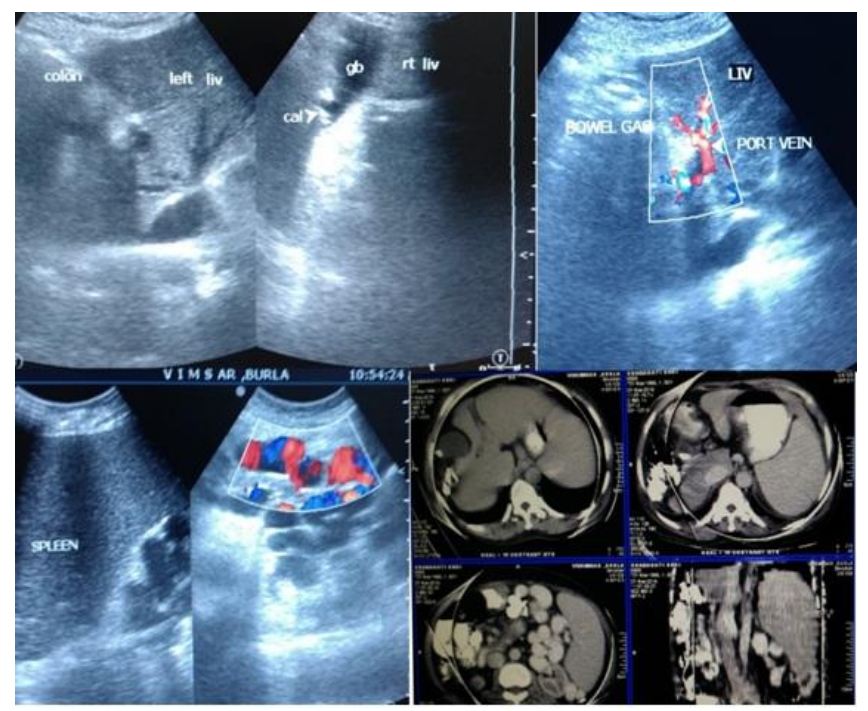

Figure 11- A Rare Case of Portal Venous Hypertension With Small Right lobe of Liver, Splenomegaly, SR Varices, high up GB, associated cholelithiasis, Confirmed by CT Scan as "Agenesis of Segment 5 \& 8 of Liver". 


\section{DISCUSSION}

Portal hypertension is classified according to the site of obstruction to the blood flow as prehepatic. Hepatic and post-hepatic. Pre-hepatic causes include portal vein occlusion, splenic vein block: Splanchnic arterio venous malformation. Hepatic causes can be presinusoidal and sinusoidal. Noncirrhotic portal fibrosis (NCPF) is a presinusoidal cause, affecting adolescents and young adults. It is due to obliterative portal venopathy resulting in portal hypertension. It is relatively less common cause of portal hypertension occurring in 3-5\% of all patients with portal hypertension worldwide, but in India it accounts for 15- $20 \%$ of cases of portal hypertension ${ }^{[3,4]}$.

The most common sinusoidal cause of obstruction to the portal blood flow is cirrhosis, which represents the final common result of a variety of insults to the liver. All types of cirrhosis lead to portal hypertension by causing obstruction to the portal flow. Portal flow is diverted into collaterals and some is directly shunted into hepatic venous radicles in the fibrous septa of the sinusoids. Regardless of the etiology of cirrhosis, the end point of this pathologic process is fibrosis with architectural distortion and formation of regenerative nodules. The induction of fibrosis occurs with activation of hepatic stellate cells, resulting in the formation of increased amounts of collagen and other components of the extracellular matrix. This results in a loss of normal hepatocytes and thus function resulting in alteration of blood flow.

Post - hepatic causes include Inferior vena cava obstruction, hepatic vein obstruction and cardiac diseases.

In our study, we were able to ascertain the etiological cause of portal hypertension in $94 \%$ of our patients, Cirrhosis is being the most common cause $(71 \%)$ for portal hypertension in our study (eastern Indian) population. Majority of the patients were middle aged males possibly due to higher consumption of alcohol leading to cirrhosis and portal hypertension.
On color Doppler normal portal vein exhibits a monophasic, low-velocity flow, with slight respiratory variation ${ }^{[5,6]}$. In normal individuals the portal vein diameter can vary from $<13 \mathrm{~mm}$ in quiet respiration to $16 \mathrm{~mm}$ in deep inspiration, as measured where the portal vein crossed anteriorly to the inferior vena cava. Bolondi ${ }^{[7]}$, Zoli ${ }^{[8]}$ and Kurol [9] all found in their respective studies that an enlarged portal vein was present in cases of portal hypertension. In 1984, La fortune found in his study that dilated portal vein was not diagnostic of portal hypertension ${ }^{[10]}$. He correlated his findings with angiography to confirm his data. Bradley Koslin in his study also found that diameter alone was not diagnostic of portal hypertension ${ }^{[11]}$. Enlarged portal vein diameter has high specificity but low sensitivity for diagnosis of portal hypertension. While it is true that an unusually large portal vein is a reliable sign of portal hypertension, it is also unfortunately true that a normal-sized portal vein in no way excludes the diagnosis ${ }^{[12]}$. Extensive review of literature conducted by Van Leeven also confirmed that diameter of portal vein was not a diagnostic criteria for portal hypertension ${ }^{[13]}$. In our study dilated portal vein was noted in $61 \%$ of cases. In normal individuals the calibre of the portal vein changes from 20-200\% between phases of respiration. Zoli in his study found that the respiratory variation in the portal vein calibre is reduced in portal hypertension ${ }^{[8]}$.The average variation between inspiration and expiration was less than $20 \%$ in portal hypertensives, and the sensitivity of this sign in diagnosing portal hypertension was $82 \%{ }^{[14]}$. Similar results were seen in our study. Loss of respiratory phasicity of portal vein was noted in $84 \%$ cases. Possible explanation is, as elevated portal pressure maximizes venous distension, so little or no additional distension will occur when the portal vein outflow is indirectly restricted by sustained inspiration. ${ }^{[12]}$

The velocity in the portal vein is approximately 15 $18 \mathrm{~cm} / \mathrm{sec}$ with a lot of variation in the range. The velocity decreases in cases where there is increased resistance to the portal blood flow as postulated by Patriquin and Bradley Koslin ${ }^{[15,11]}$. However in our 
study no significant association with reduced mean velocity was noted, only $44 \%$ cases showed decreased value. The mean PV velocity may vary depending on the presence and location of spontaneous shunts. The velocity tends to increase in the presence of a patent paraumbilical vein and decrease in the presence of splenorenal collaterals.

La Fortune and found that hepatofugal flow is an absolute sign of portal hypertension with a sensitivity of $85 \%$ and specificity of $100 \%{ }^{[10]}$. In our study only 4 had hepatofugal flow which is similar to study by Takayaso's, where 2 cases had hepatofugal flow among 80. According to him, reversal of flow in the portal vein is rare in the absence of surgical shunts ${ }^{[16]}$ Alexandra Von et al., found direction of portal vein flow was normal in $73 \%$, hepatofugal in $9 \%$ and bidirectional in $6 \%$ patients ${ }^{[17]}$, similar to our study. Hepatofugal flow not a common finding but its presence is an absolute sign of portal hypertension and very well correlates with severity of disease.

Knowing that portal vein cross-sectional area typically increases and portal velocity typically decreases in the setting of portal hypertension has led some investigators to study the ratio of these parameters, assuming that it will increase dramatically with portal hypertension. Congestion index was introduced by Moriyasu et al (1986) ${ }^{[18]}$ to address counter current changes of sectional area and blood flow in portal hypertension. They showed that the congestion index was 2.5 times higher in patients with cirrhosis and portal hypertension than in normal subjects. Haag $K$ et al (1999) ${ }^{[19]}$ concluded that the "congestion index" above 0.1 suggests the diagnosis of portal hypertension with a 95\% sensitivity and specificity. In our study $92.5 \%$ patients showed congestion index $\geq 0.1$ and only $7.5 \%$ had CI of $<0.1 \quad$ suggesting significant association between portal hypertension and CI .

Portal vein occlusion is principally caused by thrombosis (bland thrombus), tumour invasion and compression. Thrombosis may be precipitated by stagnant portal flow in patients with cirrhosis or can be a cause of portal hypertension. Other causes include hypercoagulable states, surgery, and intraperitoneal inflammatory processes, such as pancreatitis and appendicitis. In our study, 18 cases of portal vein thrombosis was found, of which 10 cases had complete thrombosis \& 8 had partial thrombosis.

In our study splenomegaly was noted in $93 \%$ of cases. La Fortune in his series found splenomegaly in $80 \%$ cases ${ }^{[10]}$. is a non-specific finding but is frequently seen in portal hypertension. In our study $85 \%$ cases shows ascites, majority having moderate ascites.

Detection of abnormal collateral vessels appears to be one of the most sensitive (70-83\%) and specific sonographic signs for the diagnosis of portal hypertension. ${ }^{[20]}$ In our study, portosystemic collaterals were visualised in $92 \%$ of the cases. Most frequent collaterals visualised were the splenorenal collaterals which were seen in $87 \%$ of cases. GE junction collaterals and paraumbilical veins were seen in 56 and $42 \%$ of cases respectively. The increased frequency of Splenorenal may be due to their easier detection because of their location and small GEJ collaterals which were not detected by ultrasonography.

Thus, most of the findings in the study were found to correlate with the previous studies related to portal hypertension.

\section{LIMITATIONS}

There are still drawbacks in assessment of portal hypertension using ultrasonography, like lack of standard scoring system for intercommunication between observers, inter observer variability, inter machine variability, interference caused by obesity, bowel gas, different states of fasting, and limitation of abdominal ultrasound to assess collaterals like haemorrhoids, retroperitoneal collaterals and esophageal varices. Another limitation of the present study was that diagnosis of portal hypertension was based on the combination of clinical, endoscopic and US findings. Objective measurements were not done to prove the diagnosis. 


\section{CONCLUSION}

Portal hypertension is a commonly encountered clinical condition with multiple causes and several sequelae. The various spectrums of findings of portal hypertension, flow metric changes and portosystemic collaterals can be accurately studied using duplex ultrasonography. Though Portal vein diameter and velocity alone were nonsignificant, loss of respiratory phasicity, congestion index, portosystemic collaterals and presence of splenomegaly and ascites shows significant association with portal hypertension. Hepatofugal flow and presence of portosystemic collaterals are diagnostic of portal hypertension. Most importantly Ultrasonography is a reliable investigation for assessing etiology of portal hypertension as we can diagnose causes like cirrhosis, portal vein occlusion or Budd-Chiari syndrome accurately by ultrasound.

\section{REFERENCES}

1. Rumack CM, Wilson SR, Charboneau JW , Levine D. The Liver .In:Wilson SR, WithersCE, editors. Diagnostic Ultrasound, $4^{\text {th }}$ ed. Philadelphia: Elsevier Mosby; 2011. P78-145.

2. Fernandez Perez FJ, García Montes JM, Castro Laria L, Martin Guerrero JM, Jimenez Saenz M, Herrerías Gutierrez JM.Usefulness of ultrasonography in the diagnosis of portal hypertension.RevEspEnferm Dig. 1998 Nov;90(11):806-812.

3. Sama SK, Bhargawa S, Gopi $\mathrm{N}$ et al. Non cirrhotic portal fibrosis.[4] Am J Med. 1971; 51: 160-69.

4. Sarin SK, Sachdev G. Nanda R. Follow up of patients after [5]variceal eradication. A comparison of patients with cirrhosis, noncirrhotic portal fibrosis and extrahepatic obstruction. Ann Surg. 1986: 204; 78-82.

5. Gorg C, Riera-Knorrenschild J, Dietrich J. Pictorial review: Color [8]Doppler ultrasound flow patterns in the portal venous system. Br J Radiol. 2002;75:919-29.
6. Owen C, Meyers P. Sonographic evaluation of the portal and [9] hepatic systems. J Diag Med Sonography. 2006; 22(5)317-28.

7. Bolondi L, Mazziotti A, Arienti V. Ultrasonographic study of portal [10]venous system in portal hypertension and after portosystemic shunt operations. Surg. 1984; 95(3):261-69.

8. Zoli M, Dondi C, Marchesini G, Cordiani MR, Melli A, Pisi E. Splanchnic vein measurements in patients with liver cirrhosis: A case control study. J Ultrasound Med. 1985; 4:641.

9. Kurol M, Forsberg L. Ultrasonographic investigation of respiratory influence on diameters of portal vessels in normal subjects. Acta Radiol Diagn (Stockh). 1986; 27:675.

10. LaFortune M, Marleau D, Breton G. Portal venous system [13] measurements in portal hypertension. Radiol. 1984; 151:27.

11. Koslin B. Duplex Doppler in portal hypertension. [14] Semin - Ultrasound and CT, MR. 1992; 13:22-33.

12. Pellerito JS, Polak JF. Ultrasound assessment of the hepatic vasculature In: Middleton WD, Robinson KA, editors. Introduction to vascular ultrasonography. 6th ed. Philaelphia: Elsevier Saunders;2012.P495-516.

13. Leeven V. Doppler ultrasound: Clinical applications. Radiol. 1990; 174:309-19.

14. Yazdi HR, Khalilian MR. Sonograhic assessment of respiratory variations in diameter of portal and splenic veins in cirrhotic patients and healthy controls. Iran $J$ Radiol. 2005;2(3,4):95-98.

15. Patriquin H, Lafortune M, Burns PN, Dauzat M. Duplex Doppler examination in portal hypertension : Technique and Anatomy. AJR.1987;149:71.

16. Takayasu K, Takashi M, Musha H. Spontaneous reversal of portal blood flow demonstrated by percutaneous transhepatic 
catheterization: Report of two cases.

Gastroenterol. 1982; 82:753.

17. Herbay AV, Frieling T, Haussinger D. Color

Doppler sonographic evaluation of spontaneous portosystemic shunts and invesion of portal venous. $J$ Clin Ultrasound. 2000;28(7):332-39.

18. F. Moriyasu, et al.: Congestion index of the portal vein. AJR Am J Roentgenol. 146:7357391986

19. Haag K, et al.: Correlation of duplex sonography findings and portal pressure in 375 patients with portal hypertension. AJR Am J Roentgenol. 172:631-635 1999.

20. Vilgrain V, Lebrec D, Menu Y, Scherrer A, Nahum H. Comparison between ultrasonographic signs and the degree of portal hypertension in patients with cirrhosis. GastrointestRadiol 1990;15:21822. 